\title{
BMJ Open RADAR study: protocol for an observational cohort study to identify early warning signals on the pathways to alcohol use disorder
}

\author{
Tim Slade, ${ }^{1}$ Wendy Swift, ${ }^{1}$ Louise Mewton, ${ }^{1}$ Kypros Kypri, ${ }^{2}$ Michael T Lynskey, ${ }^{3}$ \\ Peter Butterworth, ${ }^{4}$ Joel Tibbetts, ${ }^{1}$ Stacey McCraw, ${ }^{1}$ Emily Upton ${ }^{1}$
}

To cite: Slade T, Swift W, Mewton L, et al. RADAR study: protocol for an observational cohort study to identify early warning signals on the pathways to alcohol use disorder. BMJ Open 2017;7:e018256. doi:10.1136/ bmjopen-2017-018256

- Prepublication history for this paper is available online. To view these files please visit the journal online (http://dx.doi org/10.1136/bmjopen-2017018256).

Received 16 June 2017 Accepted 23 June 2017

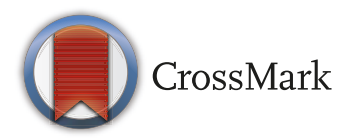

${ }^{1}$ National Drug and Alcohol Research Centre, University of New South Wales, Sydney, New South Wales, Australia

${ }^{2}$ School of Medicine and Public Health, University of Newcastle, Newcastle, New South Wales, Australia

${ }^{3}$ National Addiction Centre, Institute of Psychiatry, London, UK

${ }^{4}$ University of Melbourne, Melbourne, Victoria, Australia

Correspondence to

Dr Wendy Swift;

w.swift@unsw.edu.au

\section{ABSTRACT}

Introduction Harmful alcohol consumption, particularly alcohol use disorder (AUD), is a worldwide health priority, contributing substantially to global morbidity and mortality. The peak age of onset of AUD is 18-24, thus a deeper understanding of the young adult experience is vital if we are to identify modifiable risk factors and intervene early in the developmental course of this disabling disorder. Critical unanswered questions include: How soon after drinking initiation do AUD symptoms begin to emerge? Which symptoms come first? Do the symptoms unfold in a predictable pattern? In what ways do the emerging symptoms interact with individual, peer, family and environmental risk factors to impact on the transition to disorder?

Methods and analysis The proposed RADAR study will examine the prospective development of AUD symptoms over the young adulthood (18-24) years. We will capitalise on an existing cohort of 1911 communitybased adolescents who were recruited at age 13 and have completed a baseline and five annual follow-up assessments as part of an observational cohort study. We will interview these adolescents every 6 months between the ages of 19 and 23 to derive monthly histories of both alcohol use and AUD symptomatology, along with a comprehensive battery of risk and protective factor scales hypothesised to predict the emergence and course of AUD. The results of this study will inform the natural history of AUD and will be used to identify specific targets for prevention and early intervention of AUD.

Ethics and dissemination Ethical approval has already been granted for the study (UNSW HREC 10144). We will disseminate the results of the study through published manuscripts, conferences and seminar presentations. Data used in published manuscripts will be made available through a suitable online repository (eg, Dryad-datadryad. org).

\section{INTRODUCTION}

Globally, harmful alcohol use is a major modifiable contributor to the burden of disease. ${ }^{1}$ The typical age of initiation of alcohol use is around mid-adolescence and for a significant minority of adolescents their alcohol use will
Strengths and limitations of this study

A strength of the study is that we will be able to capture the dynamic developmental processes that characterise drinking, both moderate and problematic, during young adulthood.

- Another strength is that we will maintain total coverage of the period when the risk of developing alcohol use disorder is greatest.

- A further strength is that we will minimise recall bias for critical symptom onset information.

- Attrition bias represents the biggest threat to the validity of a cohort study.

- Study participants are currently transitioning into post-secondary school life and maintaining up-todate participant contact details will be crucial.

result in substantial harms, the most costly of which is alcohol use disorder (AUD) ${ }^{2}$ Defined as a cluster of symptoms indicating continued alcohol use despite significant problems, AUD is common and imposes a significant burden on the individual, their family and the wider society. Not only can symptoms be distressing, AUD can trigger a cascade of lifelong adverse outcomes, such as: other mental disorders, suicide, serious unintentional injury, illicit drug use, antisocial behaviour, as well as early onset of heart disease, stroke and cancer. ${ }^{3}$ While the peak age for the onset for AUD is $18-24$ years, the factors that predict the transition from alcohol use to AUD symptom onset and from symptom onset to diagnosable AUD remain largely unknown. Critical unanswered questions include: How soon after drinking initiation do AUD symptoms begin to emerge? Which symptoms come first? Do the symptoms unfold in a predictable pattern? In what ways do the emerging symptoms interact with individual, peer, family and environmental risk factors to predict the transition to disorder? Described 
herein is a protocol for the RADAR study, a cohort study of the developmental course of AUD across adolescence and young adulthood, which aims to address these unanswered questions.

The uninterrupted development of a disorder from the point of symptom onset to the time of recovery, remission or death, is referred to as its natural history. Understanding a condition's natural history is fundamental to epidemiology and has been ranked alongside understanding causality in disease prevention. ${ }^{4}$ The development of effective prevention, early intervention and treatment programmes hinges on an understanding of natural history. Only a rudimentary understanding of the natural history of AUD has emerged, largely from studies of people receiving treatment for alcohol use problems-for example, Jellinek's pioneering 1950's studies among Alcoholics Anonymous members, Vaillant's longterm study of alcoholism and more recent research by Langenbucher in the 1990s. ${ }^{5-7}$ Studies of this kind are, however, potentially limited by the clinician's illusion ${ }^{8}$ where an inaccurate view of the nature and progression of a disorder may result from only studying the highly selected cases that reach treatment services. Epidemiological research consistently demonstrates that only one in five people with lifetime AUD has sought or received treatment for disorder ${ }^{9}$ with even lower rates among young adults. ${ }^{10}$ Thus, the patterns of symptom development postulated in clinical samples may be indicative of patterns where the endpoint is chronic, well-entrenched AUD and may not reflect the diverse pathways into alcohol problems experienced by the wider population of people with AUD.

Cross-sectional general population surveys have contributed somewhat to our understanding of the temporal progression of AUD outside of the treatment setting. Nelson $e t a l^{11}$ examined retrospectively reported patterns of symptom onset in a general population survey and found that three clusters of symptoms could be validly used to organise information about the emergence of AUD. Critically, this study revealed that people who differed in their cluster assignment were also found to differ in their subsequent risk of an AUD diagnosis, showing that there is heuristic and predictive validity in identifying clusters of AUD symptoms based on temporal sequence.

Using data from a survey of US adolescents, Harford et $a l^{12}$ reported that $38 \%$ of adolescents aged 12-17 experienced at least one AUD symptom in the past year with respondents most likely to experience tolerance to the effects of alcohol, a lot of time spent drinking and drinking in hazardous situations. Using data from the same survey, McBride and Cheng ${ }^{13}$ showed that among new-onset alcohol users the symptoms of tolerance and unsuccessful efforts to cut down were most common. Interestingly, the authors showed that the likelihood of experiencing symptoms increased over the first 9 months following the initiation of alcohol use but thereafter plateaued, highlighting that AUD symptoms develop over a matter of months, not years, making it essential to investigate the natural history of AUD early in people's drinking.

Research like that just described relies exclusively on recall over long periods of time, about when AUD symptoms began. Recall bias is a significant limitation in these studies. Systematic bias is evident in the reporting of drinking onset such that reported age of onset increases with increasing chronological age and this phenomenon is amplified in those with a particularly early age of onset. ${ }^{14}$ This phenomenon is equally applicable to the dating of AUD symptom onset. Accuracy in the dating of symptom onset, which is maximised through the prospective measurement of exposure, is vital for achieving a true picture of the natural history of AUD.

In one of only a few studies to examine the longitudinal development of AUD, Behrendt $e t a l^{15}$ used data from a sample of German adolescents to demonstrate that: (A) the peak period for the onset of AUD symptoms occurred mostly in the midadolescent years (15-17), (B) around $70 \%$ of all transitions from first alcohol use to the onset of first AUD symptom occurred within 3years of initiation, and $(\mathrm{C})$ the speed of transition from first use to certain symptoms (eg, a loss of control) was elevated in those with early onset alcohol use (ie, $<13$ years). This study provides vital clues as to the early emergence of AUD; however, sequencing of symptoms was not investigated because onset data were only collected for the first AUD symptom. Moreover, the spacing of the follow-up assessments (at 2, 4 and 8 years postbaseline) was arguably too wide to provide an accurate account of the unfolding of AUD symptoms over time. In contrast, a US longitudinal study of at-risk adolescents whose fathers committed high-range drink-driving offences did not find that loss of control, when experienced as the first AUD symptom, was significantly related to the subsequent development of AUD.$^{16}$ Instead, they showed that tolerance to the effects of alcohol, particularly among those whose parents drank at problematic levels, increased the risk of progression to AUD. While these two studies highlight the clinical value of investigating the natural history of AUD symptoms in late adolescence, particularly as it plays out against a background of risk and protective factors, inconsistent findings across studies preclude firm conclusions. Our knowledge of AUD symptom development, and thus targets for early intervention, is hampered by the absence of well-conducted prospective cohort studies.

The RADAR study responds to the urgent need for high-quality, long-term prospective cohort studies to better understand the health and other implications of adolescent drinking. ${ }^{17}$ The aim of the study is to conduct a world-first, intensive, longitudinal natural history study of the developmental course of AUD across late adolescence and young adulthood. The overarching hypothesis is that the timing, rate and order of symptom development will account for differences in the risk of developing AUD. The broad objectives of the study are to (1) prospectively measure the presence, age at onset and temporal 
unfolding of AUD symptoms and (2) determine the individual, peer, family and environmental factors that, in the presence of early symptoms, predict transition to AUD.

\section{METHODS AND ANALYSIS}

RADAR will build on an existing cohort of 1911 adolescents initially recruited for participation in another study (the Australian Parental Supply of Alcohol Longitudinal Study-APSALS) ${ }^{18}$ The APSALS cohort of 1911 parentchild dyads was recruited when the children were, on average, 13 years of age (range 12-14) to examine the impact of parental supply of alcohol on the initiation of risky drinking. Participants in the APSALS cohort were recruited from public, independent and Catholic secondary schools across three Australian states (New South Wales, Tasmania and Western Australia), ensuring a wide spread of geographic locations and socioeconomic levels. Comparison with Australian population data suggests that the sample is demographically similar to the general population, although with slightly higher socioeconomic status. ${ }^{18}$ Fifty-five per cent of the participants were boys at the baseline interview in 2010/2011. Retention rates are very high, with $95 \%$ of the original cohort completing the wave 2 survey 1 year later, $92 \%$ completing the wave 3 survey, $89 \%$ completing the wave 4 survey, $87 \%$ completing the wave 5 survey and $85 \%$ completing the wave 6 survey. The wave 7 annual survey is currently in the field (as of June 2017).

Invitations to participate in RADAR have just commenced in wave 7 of APSALS, when the participants are 19 years of age on average. In RADAR, participants will be interviewed every 6 months regarding their past 6-month alcohol use for a total of 8 assessment occasions (2017-2020). Participants will be eligible for RADAR if and when they report either: (1) frequent drinking (defined as consuming any quantity of alcohol at least 1-2 days/week over a 12-month period), or (2) semifrequent binge drinking (defined as consuming more than 4 standard drinks, ie, $40 \mathrm{~g}$ alcohol, in a single session at least 3-4 days/month over a 12-month period). Once a participant is eligible for RADAR, they will continue to be assessed for AUD symptoms at all subsequent waves.

When assessed at wave 7 of APSALS, we expect that around $40 \%$ of the cohort will meet our criteria for RADAR inclusion. We therefore expect approximately 764 young adults $\left(1911^{*} 0.4\right)$ would be drinking at levels sufficient for AUD symptom assessment in the first wave of data collection. We anticipate that when the study is complete we will have prospective AUD symptom data on approximately 1324 adolescents (assuming that, on average, 80 adolescents become additional new-onset drinkers at each 6-month wave of data collection).

Eligible participants for RADAR will complete, at each wave, a telephone diagnostic interview to assess for the presence and timing of symptoms of AUD. We will use, as our core interview, the Structured Clinical Interview for DSM-IV Research Version (SCID-IV-RV), ${ }^{19}$ the gold standard for diagnosis of mental disorders including AUD. The baseline RADAR interview will assess AUD symptoms across the participant's entire lifetime, with all subsequent interviews assessing symptoms during the 6 months prior to interview. We will use a modified version of the SCID-IV-RV which has been updated for DSM-5 and the proposed ICD-11 criteria. This modified interview will also incorporate AUD symptoms outside the classification systems that have particular salience to adolescents, for example, passing out while drunk, alcohol-related intentional and unintentional injury, alcohol-related sexual risk taking. Furthermore, based on research demonstrating that adolescents can overendorse some AUD symptom questions, ${ }^{20}$ we will administer specifically designed probing questions to ensure all symptom endorsements properly reflect the intent of the symptom criteria. Trained clinical psychologists will administer all interviews. The modified SCID has demonstrated high inter-rater reliability for adolescent alcohol diagnoses $(\mathrm{kappa}=0.94) .{ }^{21}$ Before each interview takes place, verbal consent will be obtained. All interviews will be tape-recorded and a random $10 \%$ will be double coded by an independent member of the research team to ensure reliability and minimise interviewer drift. Participants will receive a $\$ 10$ gift voucher at the completion of each of their RADAR interviews.

RADAR will also use data collected as part of APSALS on a comprehensive range of potential risk and protective factors for harmful alcohol use (see ref 18). As part of RADAR specifically, we will supplement this information with scales to assess parental history of lifetime AUD, nicotine and illicit drug use by the adolescents, personality characteristics such as behavioural disinhibition and the adolescents' experience of stressful life events.

To reiterate, the aim of RADAR is to investigate the natural history of AUD symptom development with the overarching hypothesis that the timing and rate of AUD symptom emergence will contribute to individual differences in the risk of developing AUD. This overarching hypothesis will be tested through answers to five research questions. These questions and the analytical strategies we will use to answer each question are outlined below.

1. How soon after the onset of alcohol use do the symptoms of AUD begin to emerge?

Survival analysis techniques (described below) will be used to determine the latency between the onset of regular drinking and the first AUD symptom as well as a diagnosis of AUD. This will provide estimates of the cumulative probability of experiencing each specific AUD symptom as well as a diagnosis of AUD as a function of the time since onset of alcohol use.

2. What is the course of AUD once the initial symptoms are experienced?

Growth mixture modelling (GMM, described below) will be used to capture a dynamic picture of unfolding symptom trajectories over the course of adolescence. These techniques will allow us to explain the undoubtedly 
heterogeneous experience of AUD symptom development via a relatively small number of trajectories.

3. Are there predictable sequences in which adolescents experience AUD symptoms?

We will determine whether there are predictable sequences in which AUD symptoms emerge throughout young adulthood. We will use social sequence analysis ${ }^{22}$ to identify the fit to the data of different cumulative sequential models of AUD symptom progression to identify if some sequences are occurring more often than would be expected by chance. Given the multitude of possible sequence patterns, we will focus our efforts on those symptoms identified as most prevalent.

4. What individual, peer, family and environmental factors predict both the transition from alcohol use to symptom onset and the subsequent course of AUD?

This question will combine AUD symptom information with risk factor information to determine which factors are related to the onset of first AUD symptoms and the trajectories of symptom development. Based on contemporary causal models, ${ }^{23}$ we will focus our analyses particularly on factors such as sex, parental history of AUD, patterns of adolescent alcohol use, other drug use, age of alcohol use onset, subjective response to alcohol, peer alcohol use, pre-existing personality traits such as impulsivity and mental disorders. We will use logistic regression to model the odds of experiencing particular first-onset AUD symptoms as a function of the risk factors. Similarly, multinomial logistic regression will be used to model the odds of being in a given longitudinal trajectory class as a function of the risk factors.

5. How does the experience of early AUD symptoms interact with individual, peer, family and environmental factors to predict the onset of AUD?

As an extension to research question 4, we will examine the synergistic effects of certain risk factors and certain first-onset symptoms in predicting the subsequent development of AUD. Working on the assumption that the onset of certain symptoms, for example, tolerance to the effects of alcohol, will increase the risk of developing AUD particularly among certain subgroups of the population (eg, those with a family history of AUD), we will use longitudinal regression techniques (eg, mixed effects regression, generalised estimating equations) to model the risk of developing AUD as a function of the interaction between individual, peer, family and environmental risk factors and the critical first-onset AUD symptoms.

Taken together these research questions will provide a comprehensive picture of the natural history of AUD symptoms, their precursors and their consequences and point to critical windows within which the risk of developing AUD is heightened.

Kaplan-Meier curves will be generated to describe survival until the emergence of the first (and subsequent) AUD symptom. Cox proportional hazards regression modelling will be used to examine the predictors of time to first AUD symptom and patterns of symptom trajectories. Using the Peduzzi et $a l^{24}$ method, the required sample size for Cox proportional hazards regression is given by the formula $n=10 * \mathrm{k} / \mathrm{p}$, where $\mathrm{k}$ is the number of regression predictors and $\mathrm{p}$ is the proportion experiencing the outcome. A reasonable estimate of $\mathrm{k}$ for any one model is 10. Given the findings presented earlier, ${ }^{25}$ a conservative estimate of $p$, the proportion experiencing any symptom of AUD in a given year, is 0.38 , giving a required sample size of $10 * 10 / 0.38=263$. Even if only $60 \%$ of eligible adolescents complete the SCID interview, we will have diagnostic data on $458(764 * 0.6)$ young adults by the end of the first wave of data collection and on $794(1324 * 0.6)$ by the end of the study. Survival analyses will be carried out using STATA V.12. Trajectories of AUD symptoms will be examined through GMM. ${ }^{26}$ This technique combines growth modelling with latent class analysis to characterise heterogeneous developmental patterns into discrete classes that collectively explain variability. The optimal number of classes can be reliably determined with traditional fit indices with sample sizes of around $600-700^{27}$. To identify predictors of trajectory class membership, multinomial logistic regression analyses within the GMM framework will be used. Based on a formula described in Muthén and Muthén, ${ }^{28}$ and assuming a moderate $( \pm 0.2)$ predictor effect, sample sizes greater than 300 are recommended. These analyses will be carried out using MPlus V.7.

\section{ETHICS AND DISSEMINATION}

Ethical approval has already been granted for the study (UNSW HREC 10144). To enable the timely dissemination of the findings, we have planned this project so as to acquire particular data that will allow us to answer important research questions at early (research question 1), intermediate (research questions 4 and 5) and late (research questions 2 and 3) stages of the project. We will disseminate the results of the study through published manuscripts, conferences and seminar presentations. Data used in published manuscripts will be made available through a suitable online repository (eg, Dryaddatadryad.org). RADAR welcomes use of the data by interested collaborators. Access will be negotiated on a case-by-case basis and a memorandum of understanding will be agreed upon by all parties.

\section{DISCUSSION}

From a public health perspective, the results will provide evidence on which modifiable factors increase risk for the onset and development of AUD. The detailed and closely spaced timing of assessments means that we will be able to accurately identify which aspects of problematic drinking emerge first and when, in the developmental course of adolescent drinking, those aspects emerge. This approach to measuring both timing and rate of symptom development is consistent with recent recognition that certain prevention and early intervention efforts may be most effective during specific developmental windows. ${ }^{29}$ 
Determining the power of certain first-onset AUD symptoms to predict the later development of AUD will fill a gap in the literature and will have implications for the development of effective early intervention programme. It is likely that different AUD symptoms have different prognostic implications and thus knowledge of which symptoms are likely to develop first, or knowledge that a particularly risky symptom has developed, can be used as an early warning signal for the progression to AUD. From a nosological perspective, the natural history of AUD represents a fundamental piece of the diagnostic validity puzzle. ${ }^{30}$ Uncovering a reliable and orderly progression of symptoms would advance the construct validity of the AUD diagnosis. Conversely, a less ordered pattern of symptoms will bring into question the existing diagnostic systems.

Contributors TS, WS, LM, KK, MTL and PB all contributed significantly to the planning, conception, design and successful funding of the RADAR study. JT, SM and EU made further contributions to the design and planned assessment methodologies to be used in the RADAR study and are contributing significantly to the acquisition of data. TS, WS and LM drafted the initial version of this manuscript. KK, MTL, PB, JT, SM and EU revised the draft critically for important intellectual content. All authors gave final approval for this version of the manuscript to be submitted for publication.

Funding This work was supported by an Australian National Health and Medical Research Council (NHMRC) Project Grant number APP1105521.

Competing interests All authors have completed the ICMJE uniform disclosure form at www.icmje.org/coi_disclosure.pdf and declare: financial support for the submitted work from the Australian National Health and Medical Research Council (NHMRC APP1105521); no financial relationships with any organisations that might have interest in the submitted work in the previous 3 years; no other relationships or activities that could appear to have influenced the submitted work.

Ethics approval University of New South Wales Human Research Ethics Committee.

Provenance and peer review Not commissioned; peer reviewed for ethical and funding approval prior to submission.

Open Access This is an Open Access article distributed in accordance with the Creative Commons Attribution Non Commercial (CC BY-NC 4.0) license, which permits others to distribute, remix, adapt, build upon this work non-commercially, and license their derivative works on different terms, provided the original work is properly cited and the use is non-commercial. See: http://creativecommons.org/ licenses/by-nc/4.0/

(c) Article author(s) (or their employer(s) unless otherwise stated in the text of the article) 2017. All rights reserved. No commercial use is permitted unless otherwise expressly granted.

\section{REFERENCES}

1. Rehm J, Mathers C, Popova S, et al. Global burden of disease and injury and economic cost attributable to alcohol use and alcohol-use disorders. Lancet 2009;373:2223-33.

2. Collins DJ, Lapsley HM. The costs of tobacco, alcohol and illicit drug use to australian society in 2004/05. Canberra: Australian Commonwealth Department of Health and Ageing, 2008.

3. Schuckit MA. Alcohol-use disorders. Lancet 2009;373:492-501.

4. Bhopal RS. Concepts of Epidemiology. Oxford University Press: Oxford, 2002.

5. Jellinek EM. Phases of alcohol addiction. Q J Stud Alcohol 1952;13:673-84.
6. Vaillant GE. The natural history of alcoholism Revisited. Cambridge Massachusetts: Harvard University Press, 1995.

7. Langenbucher JW, Chung T. Onset and staging of DSM-IV alcohol dependence using mean age and survival-hazard methods. $J$ Abnorm Psychol 1995;104:346-54.

8. Cohen P, Cohen J. The clinician's illusion. Arch Gen Psychiatry 1984;41:1178-82.

9. Teesson M, Hall W, Slade T, et al. Prevalence and correlates of DSM IV alcohol abuse and dependence in Australia: findings of the 2007 NSMHWB. Addiction 2010;105:2085-94.

10. Mewton L, Teesson M, Slade T, et al. The epidemiology of DSMIV alcohol use disorders amongst young adults in the australian population. Alcohol Alcohol 2011;46:185-91.

11. Nelson CB, Little RJ, Heath AC, et al. Patterns of DSM-III-R alcohol dependence symptom progression in a general population survey. Psychol Med 1996;26:449-60.

12. Harford $\mathrm{T}, \mathrm{Yi} \mathrm{H}$, Faden $\mathrm{V}$, et al. The dimensionality of DSM IV Alcohol Use Disorders among adolescents. Alcoholism: Clinical and Experimental Research 2009;33:868-78.

13. McBride O, Cheng HG. Exploring the emergence of alcohol use disorder symptoms in the two years after onset of drinking. Addiction 2010;106:555-63.

14. Sartor CE, Bucholz KK, Nelson EC, et al. Reporting Bias in the association between age at first alcohol use and heavy episodic drinking. Alcohol Clin Exp Res 2011;35:no-25.

15. Behrendt S, Wittchen HU, Höfler M, et al. Risk and speed of transitions to first alcohol dependence symptoms in adolescents: a 10-year longitudinal community study in Germany. Addiction 2008;103:1638-47.

16. Buu A, Wang W, Schroder SA, et al. Developmental emergence of alcohol use disorder symptoms and their potential as early indicators for progression to alcohol dependence. J Abnorm Psychol 2012;121:897-908.

17. McCambridge J, McAlaney J, Rowe R. Adult consequences of late adolescent alcohol consumption: a systematic review of cohort studies. PLoS Med 2011;8:e1000413.

18. Aiken A, Wadolowski M, Bruno R, et al. Cohort profile: the australian parental supply of Alcohol Longitudinal Study (APSALS). Int J Epidemiol 2015.

19. First MB, Spitzer RL, Williams JB. Structured clinical interview for DSM-IV Axis I disorders SCID-I: clinician version. administration booklet: American Psychiatric Pub 1997.

20. Slade T, Teesson M, Mewton L, et al. Do young adults interpret the DSM diagnostic criteria for alcohol use disorders as intended? a cognitive interviewing study. Alcohol Clin Exp Res 2013;37:1001-7.

21. Martin CS, Pollock NK, Bukstein OG, et al. Inter-rater reliability of the SCID alcohol and substance use disorders section among adolescents. Drug Alcohol Depend 2000;59:173-6.

22. Abbott A. Sequence analysis: new methods for old ideas. Annu Rev Sociol 1995;21:93-113.

23. Koob GF. Theoretical frameworks and mechanistic aspects of alcohol addiction: alcohol addiction as a reward deficit disorder. Curr Top Behav Neurosci 2013;13:3-30.

24. Peduzzi P, Concato J, Feinstein AR, et al. Importance of events per independent variable in proportional hazards regression analysis. $J$ Clin Epidemiol 1995;48:1503-10.

25. Harford TC, Grant BF, H-y Y, et al. Patterns of DSM-IV alcohol abuse and dependence criteria among adolescents. Alcoholism: Clinical \& Experimental Research 2005;29:810-28.

26. Muthen B, Asparouhov T. Growth mixture modeling. NW: Taylor and Francis Group, 2009.

27. Tofighi D, Enders CK. Identifying the correct number of classes in growth mixture models. In: Hancock GR, Samuelsen KM, eds. Advances in latent variable mixture models. Charlotte, NC: information Age Publishing, 2008.

28. Muthén LK, Muthén BO. How to use a Monte Carlo Study to decide on Sample size and Determine Power. Structural Equation Modeling: A Multidisciplinary Journal 2002;9:599-620.

29. Catalano RF, Fagan AA, Gavin LE, et al. Worldwide application of prevention science in adolescent health. Lancet 2012;379:1653-64.

30. Robins E, Guze SB. Establishment of diagnostic validity in psychiatric illness: its application to schizophrenia. Am J Psychiatry 1970;126:983-7. 
Correction: RADAR study: protocol for an observational cohort study to identify early warning signals on the pathways to alcohol use disorder

Slade T, Swift W, Mewton L, et al. RADAR study: protocol for an observational cohort study to identify early warning signals on the pathways to alcohol use disorder BMJ Open 2017;7:e018256. DOI:10.1136/bmjopen-2017-018256.

This article was previously published with an error.

In the methods and analysis section the text reads:

Participants will be eligible for RADAR if and when they report either: (1) frequent drinking (defined as consuming any quantity of alcohol at least 1-2 days/week over a 12-month period), or (2) semifrequent binge drinking (defined as consuming more than 4 standard drinks, ie, $40 \mathrm{~g}$ alcohol, in a single session at least $3-4$ days/month over a 12-month period).

However, the correct text should read:

Participants will be eligible for RADAR if and when they report either: (1) frequent drinking (defined as consuming any quantity of alcohol at least 1-2 days/week over a 12-month period), or (2) semifrequent binge drinking (defined as consuming more than 4 standard drinks, ie, $40 \mathrm{~g}$ alcohol, in a single session at least $2-3$ days/month over a 12-month period).

Open access This is an open access article distributed in accordance with the Creative Commons Attribution Non Commercial (CC BY-NC 4.0) license, which permits others to distribute, remix, adapt, build upon this work non-commercially, and license their derivative works on different terms, provided the original work is properly cited, appropriate credit is given, any changes made indicated, and the use is non-commercial. See: http://creativecommons.org/licenses/by-nc/4.0/.

C Author(s) (or their employer(s)) 2019. Re-use permitted under CC BY-NC. No commercial re-use. See rights and permissions. Published by BMJ.

BMJ Open 2019;9:e018256corr1. doi:10.1136/bmjopen-2017-018256corr1

Check for updates 